Supplementary Information

\title{
Synthesis and spectroscopic properties of a prototype single molecule dual imaging agent comprsing a heterobimetallic rhenium-gadolinium complex
}

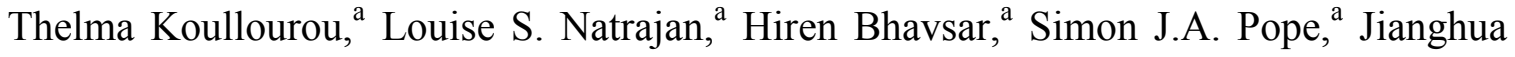
Feng, ${ }^{a}$ Johanna Narvainen, ${ }^{\mathrm{a}}$ Rachel Shaw, ${ }^{\mathrm{a}}$ Emma Scales, ${ }^{\mathrm{a}}$ Risto Kauppinen, ${ }^{\mathrm{b}}$ Alan M. Kenwright $^{\mathrm{c}}$ and Stephen Faulkner ${ }^{\mathrm{a}, *}$

a) University of Manchester, Oxford Road, Manchester, M13 9PL, UK

b) Department of Radiology, Dartmouth Medical School, 706 Vail, HB 7785 Hanover NH 03755

c) Department of Chemistry, University of Durham, South Road, Durham, DH1 3LE, UK.

\section{Luminescence spectroscopy}

Luminescence spectra of europium and rhenium derivatives were measured On a PerkinElmer LS55 fluorimeter. In the case of the ytterbium complexes, the sample was excited using a pulsed nitrogen laser (PTI-3301-337nm). Light emitted at right angles to the excitation beam was focussed onto the slits of the monochromator (PTI120), which was used to select the appropriate wavelength. The growth and decay of the luminescence at selected wavelengths was detected using a germanium photodiode (Edinburgh Instruments, EI-P) and recorded using a digital oscilloscope (Tektronix TDS220) before being transferred to the computer for analysis. Luminescence lifetimes were obtained by iterative reconvolution of the detector response (obtained by using a scatter) with exponential components for growth and decay of the metal centred luminescence, using a spreadsheet running in Microsoft Excel. The time resolve emission (TRES) spectrum for the complexes were obtained by measuring the growth and decay of the luminescence at each series of wavelengths.

\section{X-Ray Crystallography}

Data were collected at $150 \mathrm{~K}$ using a Bruker APEX II CCD diffractometer on station 9.8 
of the Synchrotron Radiation Source at CCLRC Daresbury Laboratory, at $0.69040 \mathrm{~A}^{\circ}$, from a silicon 111 monochromator. The structure was solved by direct methods using the program SHELXS-97.21. The refinement and all further calculations were performed using SHELXL-97. ${ }^{1}$

Table 1. Crystal data and structure refinement for HBLPY.

Identification code

Empirical formula

Formula weight

Temperature

Wavelength

Crystal system

Space group

Unit cell dimensions

Volume

Z

Density (calculated)

Absorption coefficient

$\mathrm{F}(000)$

Crystal size

Theta range for data collection

Index ranges

Reflections collected

Independent reflections

Completeness to theta $=27.50^{\circ}$

Absorption correction

Max. and min. transmission

Refinement method

Data / restraints / parameters

Goodness-of-fit on $\mathrm{F}^{2}$

Final R indices [I $>2 \operatorname{sigma}(\mathrm{I})]$

$\mathrm{R}$ indices (all data)

Largest diff. peak and hole
HBLPY

C34 H68 Br N6 Na O11

839.84

150(2) K

$0.69040 \AA$

Triclinic

P-1

$\mathrm{a}=10.112(2) \AA$ $\alpha=81.12(3)^{\circ}$.

$\mathrm{b}=10.838(2) \AA$ $\beta=79.24(3)^{\circ}$.

$\mathrm{c}=20.948(4) \AA$ $\gamma=84.00(3)^{\circ}$.

2221.6(7) $\AA^{3}$

2

$1.255 \mathrm{Mg} / \mathrm{m}^{3}$

$0.992 \mathrm{~mm}^{-1}$

896

$0.14 \times 0.09 \times 0.05 \mathrm{~mm}^{3}$

1.91 to $28.28^{\circ}$.

$-13<=\mathrm{h}<=13,-14<=\mathrm{k}<=14,-27<=\mathrm{l}<=27$

21914

$10902[\mathrm{R}(\mathrm{int})=0.0631]$

$99.0 \%$

Multi-scan

0.9248 and 0.8735

Full-matrix least-squares on $\mathrm{F}^{2}$

10902 / 0 / 487

0.991

$\mathrm{R} 1=0.0707, \mathrm{wR} 2=0.2014$

$\mathrm{R} 1=0.0842, \mathrm{wR} 2=0.2205$

0.747 and -0.766 e. $\AA^{-3}$ 


\section{NMR Spectroscopy}

All diamagnetic NMR spectra were recorded on a Bruker Avance 400 spectrometer, operating frequency $400 \mathrm{MH}_{\mathrm{z}}\left({ }^{1} \mathrm{H}\right), 125 \mathrm{MHz}\left({ }^{13} \mathrm{C}\right)$, variable temperature unit at $300 \mathrm{~K}$, unless otherwise stated. Chemical shifts are reported in parts per million and referenced to residual proton resonances in $\mathrm{CDCl}_{3}$ or $\mathrm{D}_{2} \mathrm{O}$ and calibrated externally to TMS. Proton NMR spectra of paramagnetic compounds were recorded on a Varian Inova 500, operating frequency $500 \mathrm{MHz}$ at $295 \mathrm{~K}$.

\section{1,4,7-Tris(tert-butoxycarbonylmethyl)-1,4,7,10-tetraazacyclododecane, Hydrobromide salt, $\mathbf{H B r}$ (1). Prepared according to a literature procedure ${ }^{2}$}

\section{4-[4,7,10-tris(tert-butoxycarbonylmethyl)-1,4,7,10-tetraazacyclododecan-1-}

yl]methyl pyridine.NaBr (2). To a stirred solution of the hydrobromide salt of cyclen ester $(0.459 \mathrm{~g}, 0.79 \mathrm{mmol})$ and sodium hydrogen carbonate $(0.17 \mathrm{~g}, 1.98 \mathrm{mmol})$ in acetonitrile $\left(15 \mathrm{~cm}^{3}\right)$ was added dropwise an acetonitrile solution of 4-bromomethyl pyridine $\left(0.25 \mathrm{~g}, 0.83 \mathrm{mmol}, 15 \mathrm{~cm}^{3}\right)$ over 30 minutes. The mixture was then heated gently to reflux temperature for a further 24 hours. After this time, the solution was filtered to remove the inorganic salts and the filtrate evaporated to dryness. The crude product was then subjected to column chromatography on silica; $\mathrm{CH}_{2} \mathrm{Cl}_{2}: \mathrm{MeOH} / \mathrm{NH}_{3}$, gradient from $100 / 0 / 1$ to $95 / 5 / 1$. All solvents were then removed under reduced pressure and the product recrystallised from $\mathrm{MeCN}: \mathrm{Et}_{2} \mathrm{O}$ at $-18^{\circ} \mathrm{C}$ to afford 2 as a white crystalline solid in $49 \%$ yield $(0.44 \mathrm{~g})$. $\mathrm{ES}^{+} \mathrm{MS}(\mathrm{MeCN}): \mathrm{m} / \mathrm{z} 606\{\mathrm{M}-\mathrm{H}\}^{+}(100 \%), 628\{\mathrm{M}-\mathrm{Na}\}^{+}$ (75\%). IR (solid) $v(\mathrm{C}=\mathrm{O}) 1714,1727 \mathrm{~cm}^{-1},(\mathrm{NH}) 3461 \mathrm{~cm}^{-1} . \mathrm{NMR}\left(\mathrm{CDCl}_{3}\right): \delta_{\mathrm{H}}: 1.41$, 1.40 (s, overlapping, 27H, ${ }^{t} \mathrm{Bu}$ ), $2.15-3.60$ (br, 24H, $\mathrm{CH}_{2}$ cyclen), $7.35\left(2 \mathrm{H}, \mathrm{d},{ }^{3} \mathrm{~J}_{\mathrm{HH}} 5.6\right.$ $\mathrm{Hz}, \mathrm{Ar} H), 8.48\left(2 \mathrm{H}, \mathrm{d},{ }^{3} \mathrm{~J}_{\mathrm{HH}} 5.6 \mathrm{~Hz}, \mathrm{Ar} H\right) . \delta_{\mathrm{C}}: 27.86,27.97,28.15\left(\mathrm{CCH}_{3}\right), 32.90,50.26$,

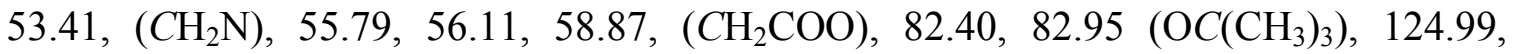
146.72, 150.15, (Ar) 173.69, $172.69(C=\mathrm{O})$. Anal. Calc for $\mathrm{C}_{32} \mathrm{H}_{55} \mathrm{~N}_{5} \mathrm{O}_{6} \cdot \mathrm{NaBr} \cdot \mathrm{H}_{2} \mathrm{O}: \mathrm{C}$, 52.89, H, 7.91, N, 9.64. Found: C, 52.72, H, 8.09, N, 9.47.

4-[4,7,10-tris(carboxymethyl)-1,4,7,10-tetraazacyclododecan-1-yl]methyl pyridine (3). 4-[4,7,10-tris(tert-butoxycarbonylmethyl)-1,4,7,10-tetraazacyclododecan-1-yl]methyl 
pyridine $(0.373 \mathrm{~g}, 1.0 \mathrm{mmol})$ was dissolved in dichloromethane $\left(5 \mathrm{~cm}^{3}\right)$ and trifluoroacetic acid $\left(5 \mathrm{~cm}^{3}\right)$ was added dropwise to the stirring solution. The reaction mixture was allowed to stir at room temperature for 24 hours. All volatiles were then removed under reduced pressure and the residue washed repeatedly with dichloromethane $\left(3 \times 10 \mathrm{~cm}^{3}\right)$ and methanol $\left(3 \times 10 \mathrm{~cm}^{3}\right)$. The resultant solid was then dissolved in ethanol $\left(7 \mathrm{~cm}^{3}\right)$ and precipitated by the slow addition of diethyl ether. The hygroscopic product was filtered, and washed with diethyl ether to afford a white solid (0.17 g, $65 \%)$. ES ${ }^{+}$ MS (MeCN): m/z $436\{\mathrm{M}-\mathrm{H}\}^{+}, 458\{\mathrm{M}-\mathrm{Na}\}^{+}$. NMR $\left(\mathrm{D}_{2} \mathrm{O}\right): \delta_{\mathrm{H}}: 2.6-4.5\left(\mathrm{br}, 24 \mathrm{H}, \mathrm{CH}_{2}\right.$ cyclen), $8.10\left(\mathrm{~d}, 2 \mathrm{H},{ }^{3} \mathrm{~J}_{\mathrm{HH}} 8.0 \mathrm{~Hz}, \operatorname{Ar} H\right), 8.65\left(\mathrm{~d}, 2 \mathrm{H},{ }^{3} \mathrm{~J}_{\mathrm{HH}} 8.0 \mathrm{~Hz} \operatorname{Ar} H\right) . \delta_{\mathrm{C}}: 48.21,48.83$, 50.48, 51.63, 53.71, 55.99, $56.62\left(\mathrm{CH}_{2}\right), 127.93,141.50$ (CH-Ar), 158.65 (C-Ar), 174.63 (CO). Anal. Calc for: $\mathrm{C}_{20} \mathrm{H}_{28} \mathrm{~N}_{5} \mathrm{O}_{6} \cdot 0.75 \mathrm{CF}_{3} \mathrm{COOH} .7 . \mathrm{H}_{2} \mathrm{O}, \mathrm{C}, 39.97, \mathrm{H}, 6.55, \mathrm{~N}, 10.84$. Found: C, 40.08, H, 6.48, N, 11.24.

\section{Preparation of complexes}

General method To a solution of the tris-acid in methanol $\left(3-5 \mathrm{~cm}^{3}\right)$ was added one equivalent of the corresponding lanthanide triflate in methanol $\left(3-5 \mathrm{~cm}^{3}\right)$. The reaction mixture was then heated gently to $40^{\circ} \mathrm{C}$ for $24 \mathrm{~h}$. After this time, all volatiles were removed under reduced pressure and the crude product recrystallised by slow diffusion of diethyl ether into an ethanolic solution of the complex $\left(\sim 5 \mathrm{~cm}^{3}\right)$ at room temperature.

\section{Ytterbium-4-[4,7,10-tris(carboxymethyl)-1,4,7,10-tetraazacyclododecan-1-yl]methyl} pyridine (Yb.3). Obtained as a brown solid (0.07 g, $72 \%$ ). MALDI MS ( $\alpha$-Methanol): $\mathrm{m} / \mathrm{z} 609\{\mathrm{M}-\mathrm{H}\}^{+}, 631$ broad clusters $\{\mathrm{M}-\mathrm{Na}\}^{+}$. IR (solid) $v(\mathrm{C}=\mathrm{O}) 1586 \mathrm{~cm}^{-1}$ (br). UV-vis $\left(\mathrm{H}_{2} \mathrm{O}\right) \lambda_{\max }=258 \mathrm{~nm}\left(\varepsilon 2630 \mathrm{M}^{-1} \mathrm{~cm}^{-1}\right)$. NMR $\left(\mathrm{D}_{2} \mathrm{O}\right): \delta_{\mathrm{H}}:-136,-101,-96,-91,-88,-84,-$ $79,-72,-64,-55,-51,-45,-39,21,22,24,26,27,29,32,34,40,41,51,72,88,91,136$, $160,190,218$. Only resolved peaks outside the range +20 to $-20 \mathrm{ppm}$ reported.

\section{Europium-4-[4,7,10-tris(carboxymethyl)-1,4,7,10-tetraazacyclododecan-1-yl]methyl} pyridine. Obtained as a white solid. (0.58g, 95\%). MALDI MS ( $\alpha$-Methanol): $\mathrm{m} / \mathrm{z}$ $1171\{2 \mathrm{M}\}(90 \%)$ broad clusters, IR (solid) $v(\mathrm{C}=\mathrm{O}) 1583 \mathrm{~cm}^{-1}$ (broad), UV-vis $\left(\mathrm{H}_{2} \mathrm{O}\right)$ 
$\lambda_{\max }=258 \mathrm{~nm}\left(\varepsilon 1770 \mathrm{M}^{-1} \mathrm{~cm}^{-1}\right) ; \delta_{\mathrm{H}}\left(\mathrm{D}_{2} \mathrm{O}\right): 34,31,30,10,8,-1,-2,-3,-4,-6,-7,-8,-11$, $-12,-14,-15,-16,-17$ (Only resolved peaks outside the range 0 to $+6 \mathrm{ppm}$ reported).

Gadolinium-4-[4,7,10-tris(carboxymethyl)-1,4,7,10-tetraazacyclododecan-1yl]methyl pyridine (Gd.3). Obtained as a white solid (0.093 g, $69.5 \%$ ). MALDI MS ( $\alpha$ Methanol): $\mathrm{m} / \mathrm{z} 1207$, broad clusters $\{2 \mathrm{M}-\mathrm{Na}\}^{+}(20 \%)$. IR (solid) $v(\mathrm{C}=\mathrm{O}) 1583 \mathrm{~cm}^{-1}$ (broad); UV-vis $\left(\mathrm{H}_{2} \mathrm{O}\right) \lambda_{\max }=258 \mathrm{~nm}\left(\varepsilon 1143 \mathrm{M}^{-1} \mathrm{~cm}^{-1}\right)$.

fac-chlorotricarbonyl(2,2'-bypyridine)rhenium(I). Prepared according to the literature procedure. $^{3}$

fac-(acetonitrile)tricarbonyl(2,2'-bypyridine)rhenium(I) triflate. According to a modification of a literature procedure, ${ }^{4}$ a solution of AgOTf $(0.256 \mathrm{~g}, 1.00 \mathrm{mmol})$ in 5 $\mathrm{cm}^{3}$ of THF was added to a solution of $f a c$-chlorotricarbonyl(bypiridine)rhenium(I) $(0.48 \mathrm{~g}, 1.00 \mathrm{mmol})$ in $50 \mathrm{~cm}^{3}$ of acetonitrile in the dark under $\mathrm{N}_{2}$ for $16 \mathrm{~h}$. The reaction was then allowed to settle and the supernatant decanted from the salts and filtered through a pad of celite to eliminate any residual $\mathrm{AgCl}$. All volatiles were removed under reduced pressure and the crude product recrystallised from $\mathrm{CHCl}_{3} / \mathrm{Et}_{2} \mathrm{O}$ at $-18^{\circ} \mathrm{C}$ to yield the title compound as yellow needles $(0.40 \mathrm{~g}, 65 \%)$. NMR $\left(\mathrm{CDCl}_{3}\right) \delta_{\mathrm{H}}: 8.93(\mathrm{dd}, 2 \mathrm{H}$,

$\left.{ }^{3} \mathrm{~J}_{\mathrm{HH}} 5.44 \mathrm{~Hz},{ }^{4} \mathrm{~J}_{\mathrm{HH}} 0.96 \mathrm{~Hz}, \mathrm{H}_{3}\right), 8.66\left(\mathrm{~d}, 2 \mathrm{H},{ }^{3} \mathrm{~J}_{\mathrm{HH}} 8.20 \mathrm{~Hz}, \mathrm{H}_{6}\right), 8.28\left(\mathrm{td}, 2 \mathrm{H},{ }^{3} \mathrm{~J}_{\mathrm{HH}} 8.12\right.$ $\left.\mathrm{Hz},{ }^{2} \mathrm{~J}_{\mathrm{HH}} 1.24 \mathrm{~Hz}, \mathrm{H}_{5}\right), 7.65\left(\mathrm{td}, 2 \mathrm{H},{ }^{3} \mathrm{~J}_{\mathrm{HH}} 5.52 \mathrm{~Hz},{ }^{4} \mathrm{~J}_{\mathrm{HH}} 1.02 \mathrm{~Hz}, \mathrm{H}_{4}\right)$. Anal. Calc for: $\mathrm{C}_{16} \mathrm{H}_{11} \mathrm{~N}_{3} \mathrm{O}_{6} \mathrm{~F}_{3} \mathrm{SRe} .0 .5 \mathrm{CHCl}_{3}, \mathrm{C}, 30.54, \mathrm{H}, 1.68, \mathrm{~N}, 6.11$. Found: C, 30.74, H, 1.57, N, 6.51 .

fac-rhenium(I)tricarbonyl-2,2'-bipyridine-ytterbium(4-[4,7,10-tris(carboxymethyl)1,4,7,10-tetraazacyclododecan-1-yl]methyl pyridine triflate (Yb.3.Re(Bpy)(CO) $)_{3}$ OTf). A solution of ytterbium-4-[4,7,10-tris(carboxymethyl)1,4,7,10-tetraazacyclododecan-1-yl]methyl pyridine (0.607 g, $1.00 \mathrm{mmol})$ in ethanol 30 $\mathrm{cm}^{3}$ was adjusted to $\mathrm{pH} 8$ by the addition of $\mathrm{NaHCO}_{3}$. A solution of $\operatorname{Re}($ bpy $)(\mathrm{CO})_{3}(\mathrm{MeCN})$.OTf $(0.62 \mathrm{~g}, 1.00 \mathrm{mmol})$ in acetonitrile $(25 \mathrm{~mL})$ was then added to this solution and the reaction mixture heated to $50^{\circ} \mathrm{C}$ and stirred for $17 \mathrm{~h}$. The solvents 
were then removed under reduced pressure and the solid washed with water to remove any unreacted starting materials. After removal of the water by evaporation, the complex was dissolved in a minimal volume of THF; slow diffusion of hexane into this solution afforded $\mathrm{Yb} .3 . \operatorname{Re}(\mathrm{bpy})(\mathrm{CO})_{3}$.OTf as a yellow solid $(0.055 \mathrm{~g}$, $55 \%)$. MALDI MS ( $\alpha$ Methanol): $\mathrm{m} / \mathrm{z} 1034$ broad cluster $\{\mathrm{M}-\mathrm{H}\}^{+}(10 \%)$. IR (solid) v: $(\mathrm{C}=\mathrm{O}) 1601$ (broad), (CO) 1873, 1890, $2013 \mathrm{~cm}^{-1}$; UV-vis $\left(\mathrm{H}_{2} \mathrm{O}\right) \lambda_{\max }=357 \mathrm{~nm}\left(\varepsilon 1068 \mathrm{M}^{-1} \mathrm{~cm}^{-1}\right)$. NMR $\left(\mathrm{D}_{2} \mathrm{O}\right)$ $\delta_{\mathrm{H}}:-103,-74,-73,-70,-62,-60,-25,-28,46,112,114,128143$, (only resolved peaks outside the range +20 to $-20 \mathrm{ppm}$ reported).

fac-rhenium(I)tricarbonyl-2,2'-bipyridine-gadolinium(4-[4,7,10tris(carboxymethyl)-1,4,7,10-tetraazacyclododecan-1-yl]methyl pyridine triflate $\left.(\mathbf{G d} \text {.3.Re(Bpy)(CO) })_{3} \mathbf{O T f}\right)$. Followed the same procedure as above, obtained as a yellow solid (0.09 g, $49 \%)$ MS ( $\alpha-\mathrm{MeOH}): \mathrm{m} / \mathrm{z} 1020$ broad cluster $\{\mathrm{M}-\mathrm{H}\}^{+}(10 \%), 1043$ broad cluster $\{\mathrm{M}-\mathrm{Na}\}^{+}(11 \%), 426\left\{\mathrm{Re}(\mathrm{bipy})(\mathrm{CO})_{3}\right\}^{+}(100 \%), 615\{\mathrm{Gd} .3\}^{+}(92 \%)$. UV-vis $\left(\mathrm{H}_{2} \mathrm{O}\right) \lambda_{\max } 367\left(\varepsilon 1198 \mathrm{M}^{-1} \mathrm{~cm}^{-1}\right)$. 
Fitted decay of the observed luminescence at $980 \mathrm{~nm}$ from $\mathrm{Yb} .3\left(\mathrm{H}_{2} \mathrm{O},, \lambda_{\mathrm{ex}}=337 \mathrm{~nm}\right.$, $\tau=1.20 \mu s)$

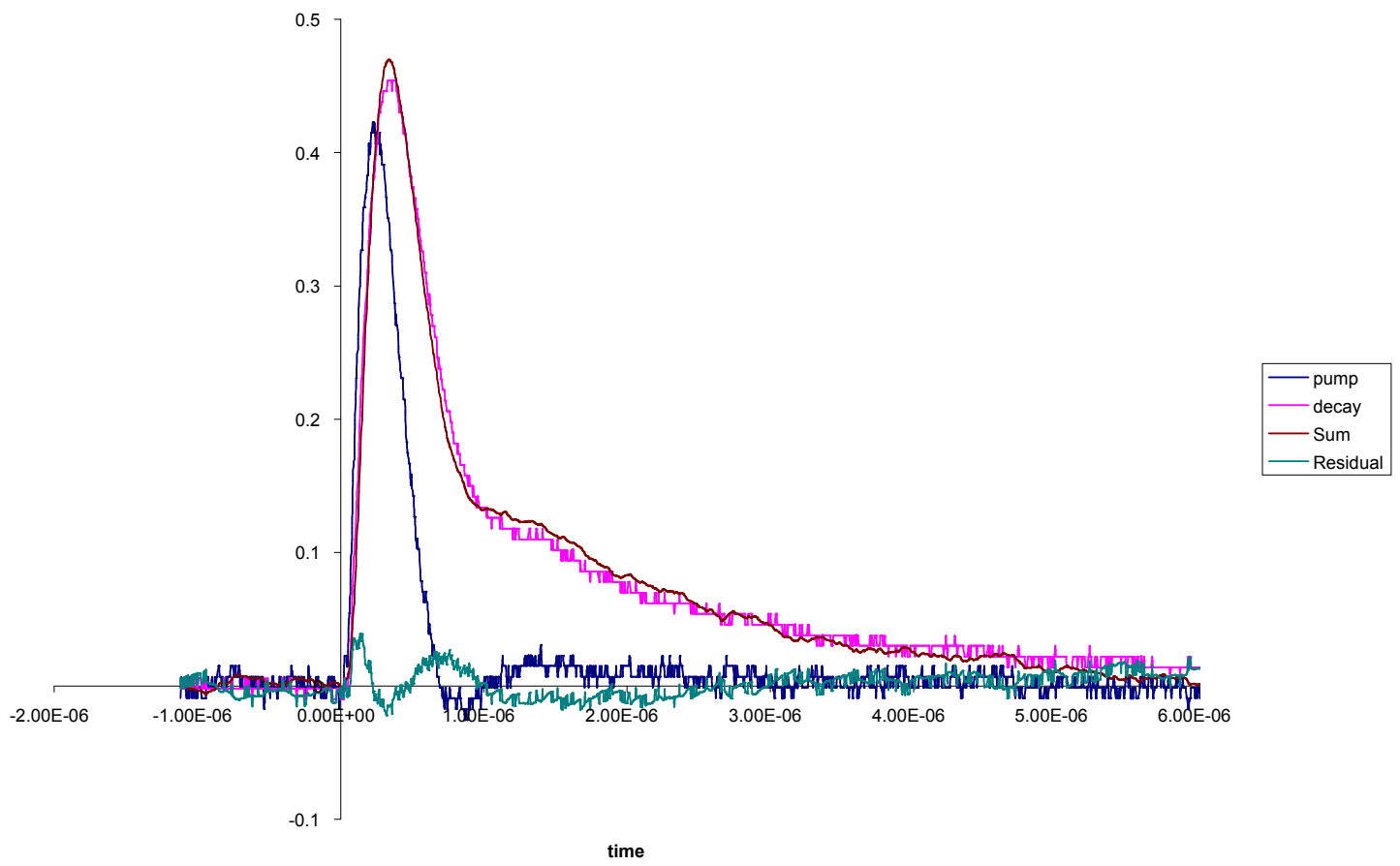

Fitted decay of the observed luminescence at $980 \mathrm{~nm}$ from $Y b .3\left(D_{2} O, \lambda_{e x}=337 \mathrm{~nm}\right.$, $\tau=5.34 \mu s)$

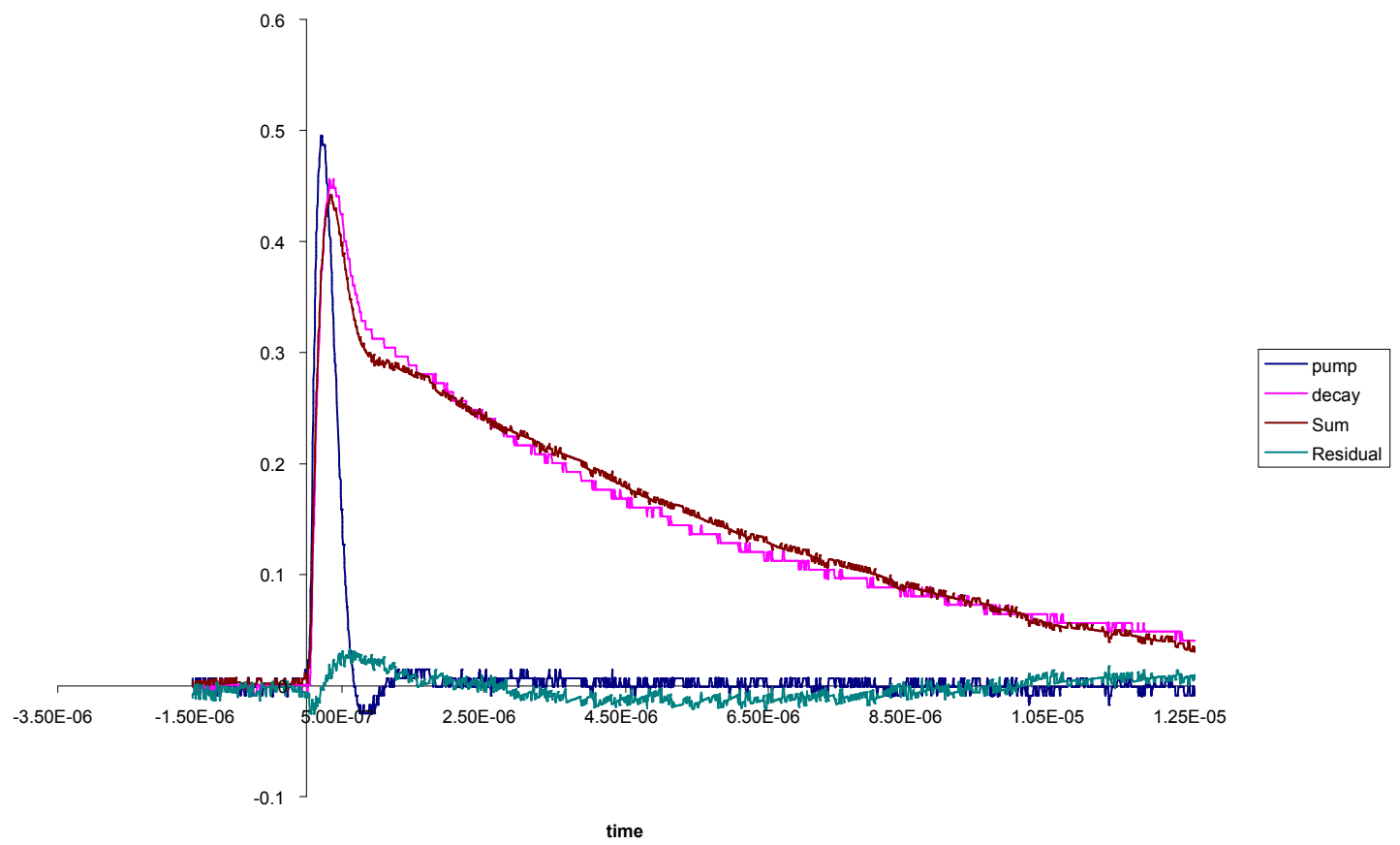


Fitted decay of the observed luminescence at $980 \mathrm{~nm}$ from $\mathrm{Yb} .3 . \operatorname{Re}(\mathrm{Bpy})(\mathrm{CO})_{3} \mathrm{OTf}$ $\left(\mathrm{H}_{2} \mathrm{O}, \lambda_{\text {ex }}=337 \mathrm{~nm}, \tau=1.47 \mu \mathrm{s}\right)$

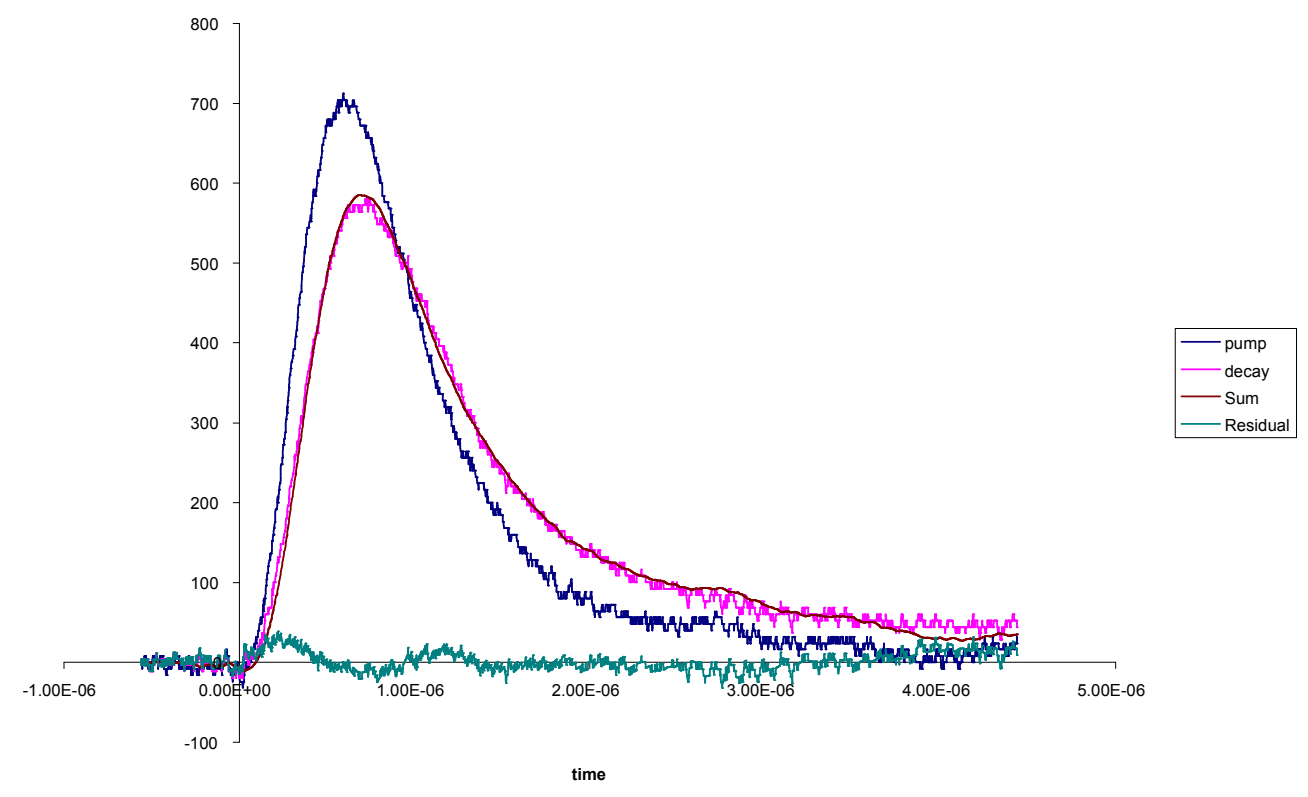

Fitted decay of the observed luminescence at $980 \mathrm{~nm}$ from $\mathrm{Yb} .3 . \operatorname{Re}(\mathrm{Bpy})(\mathrm{CO})_{3} \mathrm{OTf}$ $\left(D_{2} \mathrm{O}, \lambda_{\text {ex }}=337 \mathrm{~nm}, \tau=5.30 \mu \mathrm{s}\right)$

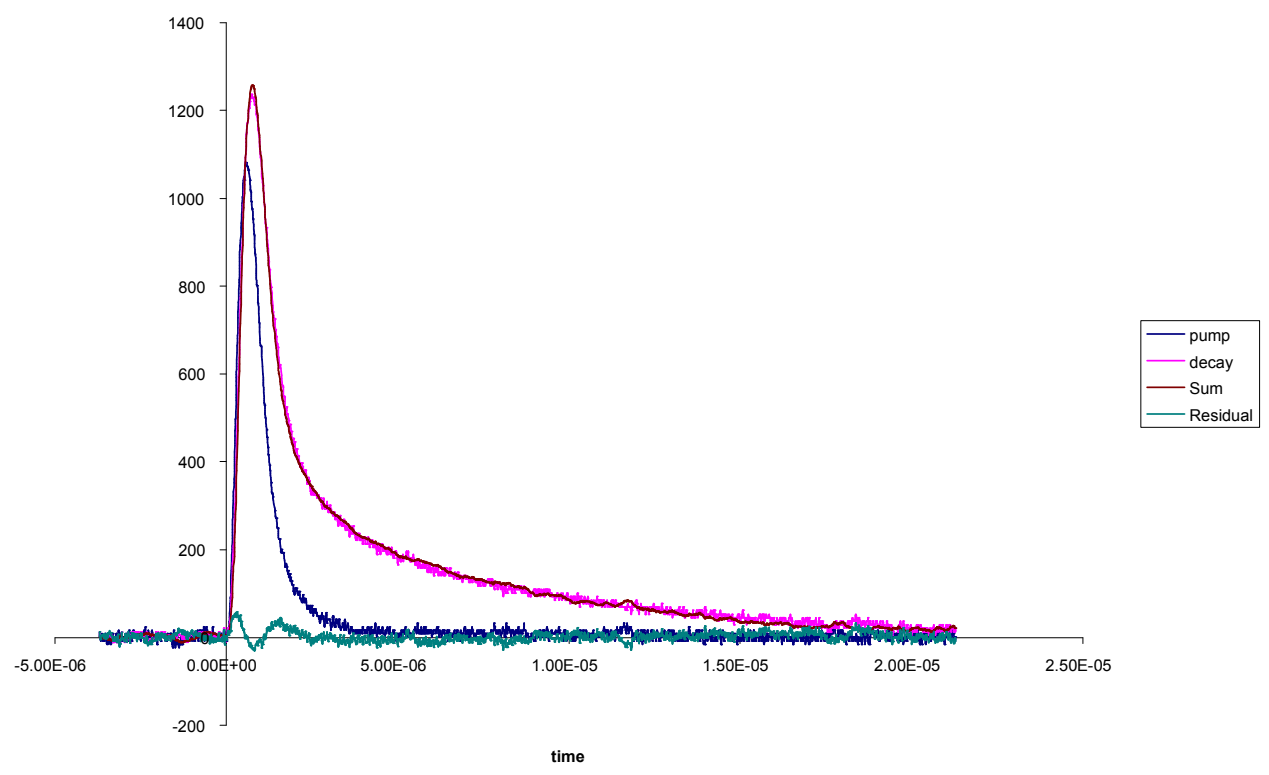


${ }^{1} \mathrm{H}$ NMR spectrum of $\mathrm{Yb.3}\left(\mathrm{D}_{2} \mathrm{O}\right)$

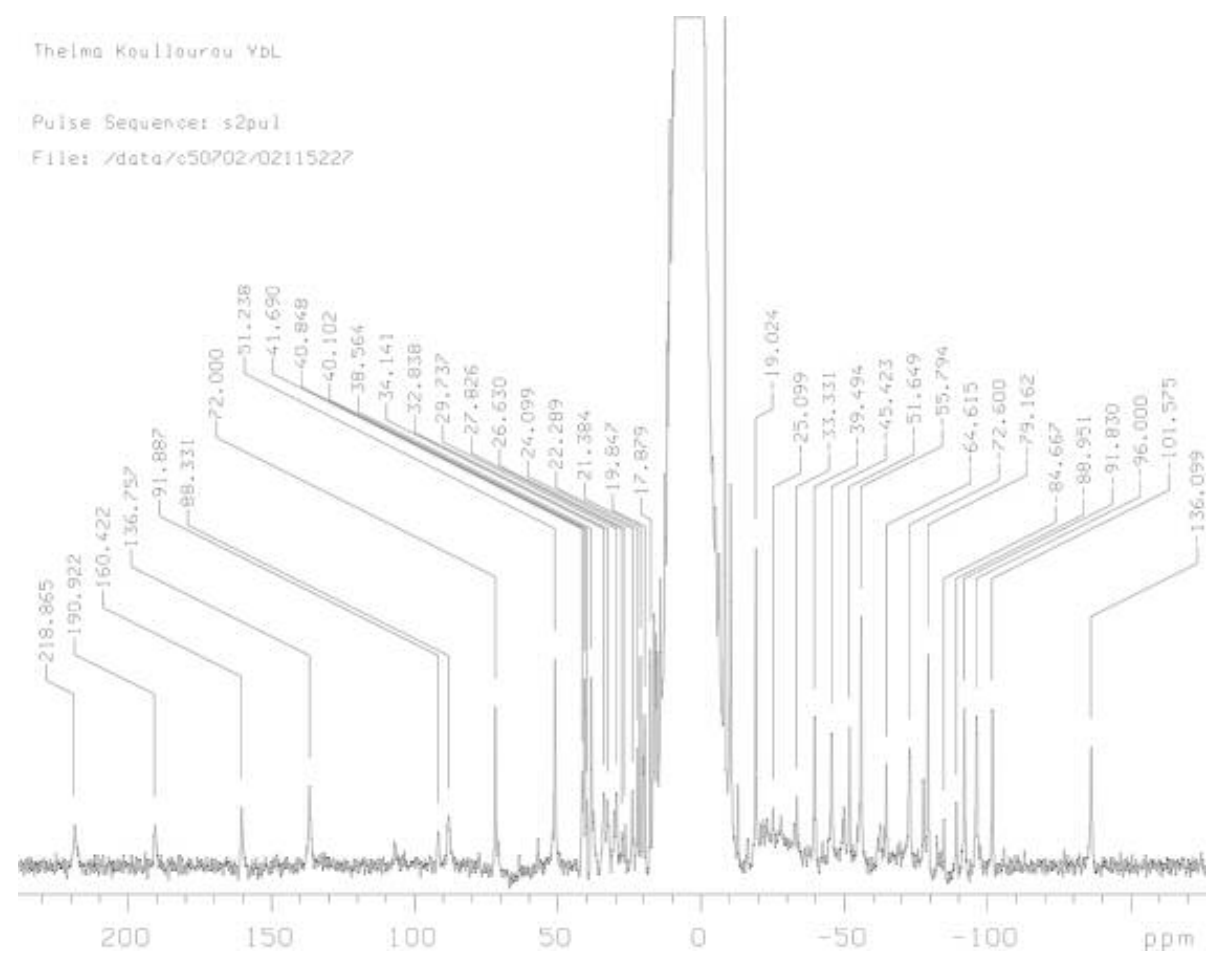

\section{${ }^{1} \mathrm{H}$ NMR spectrum of $\mathrm{Yb.3.Re}(\mathrm{Bpy})(\mathrm{CO})_{3} \mathrm{OTf}\left(\mathrm{D}_{2} \mathrm{O}\right)$}

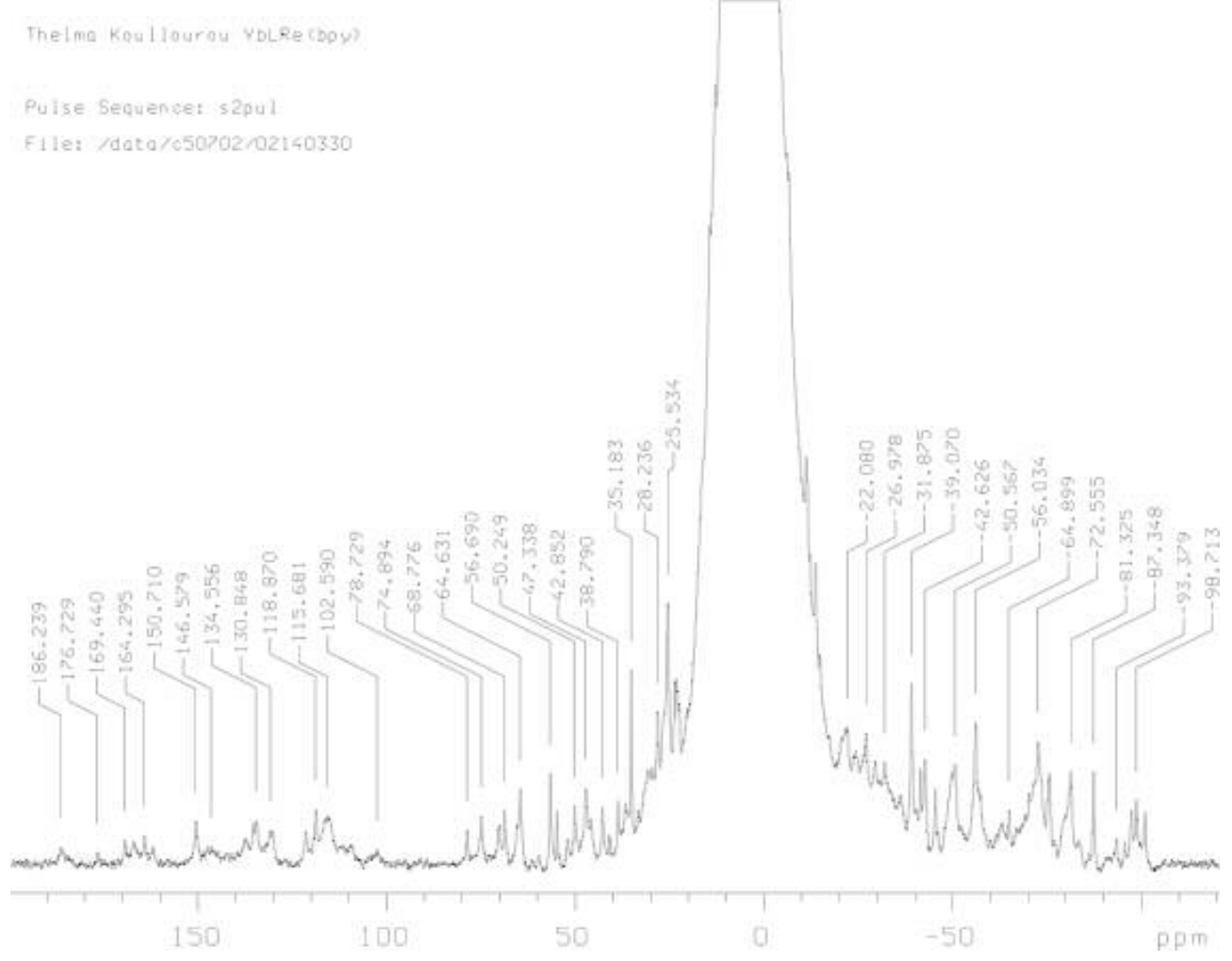




\section{Relaxivity measurements}

For a contrast agent (CA), the solvent longitudinal relaxation rate, $1 / \mathrm{T}_{1}$, is linearly dependent on the concentration of CA as described in the following equation:

$\left(1 / \mathrm{T}_{1}\right)_{\mathrm{obsd}}=\left(1 / \mathrm{T}_{1}\right)_{\mathrm{d}}+\mathrm{R}_{1} \times[\mathrm{CA}]$

Where $\left(1 / \mathrm{T}_{1}\right)_{\text {obsd }}$ and $\left(1 / \mathrm{T}_{1}\right)_{\mathrm{d}}$ are the measured solvent relaxation rate in the presence and absence of the paramagnetic species respectively. $R_{1}$, the relaxivity in units of $\mathrm{mM}^{-1} \mathrm{~s}^{-1}$, reflects the relaxation enhancement ability of the CA.

Solvent $T_{1}$ relaxation times were measured by inversion recovery, ensuring that the delay between subsequent measurements was greater than $5 \mathrm{xT}_{1} \cdot \mathrm{T}_{1}$ relaxation times were measured for several concentrations of Gd.3 and Gd.3Re(bpy)OTf in $\mathrm{H}_{2} \mathrm{O}$ and PBS (phosphate buffered saline) at $500 \mathrm{MHz}$. The relationship of relaxation rate vs. concentration was linear in each case with a correlation coefficient 0.9884 and a relaxivity of $5.83 \mathrm{mM}^{-1} \mathrm{~s}^{-1}$ for $\mathrm{Gd} .3$ in $\mathrm{H}_{2} \mathrm{O}$ and a correlation coefficient of 0.9982 and relaxivity of $4.46 \mathrm{mM}^{-1} \mathrm{~s}^{-1}$ in PBS. A correlation coefficient of 0.9970 and relaxivity of $8.62 \mathrm{mM}^{-1} \mathrm{~s}^{-1}$ was determined for Gd.3Re(bpy)OTf in $\mathrm{H}_{2} \mathrm{O}$ and a correlation coefficient of 0.9843 and relaxivity of $3.96 \mathrm{mM}^{-1} \mathrm{~s}^{-1}$ was determined in PBS. Concentrations of gadolinium were established from elemental analysis data and by volumetric methods. 
Relaxivity of $\mathrm{Gd} .3$ in $\mathrm{H}_{2} \mathrm{O}\left(500 \mathrm{MH}_{\mathrm{z}}\right)$

MW: $591.7 \mathrm{~g} / \mathrm{mol}$

\begin{tabular}{|l|l|l|}
\hline Concentration $(\mathrm{mM})$ & $\mathrm{T}_{1}(\mathrm{~s})$ & $1 / \mathrm{T}_{1}\left(\mathrm{~s}^{-1}\right)$ \\
\hline 0 & 3.0900 & 0.3236 \\
\hline 0.05 & 1.5460 & 0.6468 \\
\hline 0.1 & 1.0500 & 0.9524 \\
\hline 0.2 & 0.6488 & 1.5413 \\
\hline 0.3 & 0.4732 & 2.1133 \\
\hline 0.5 & 0.3038 & 3.2916 \\
\hline 0.7 & 0.2272 & 4.4014 \\
\hline 0.8 & 0.2228 & 4.4883 \\
\hline 1.0 & 0.1526 & 6.5531 \\
\hline
\end{tabular}

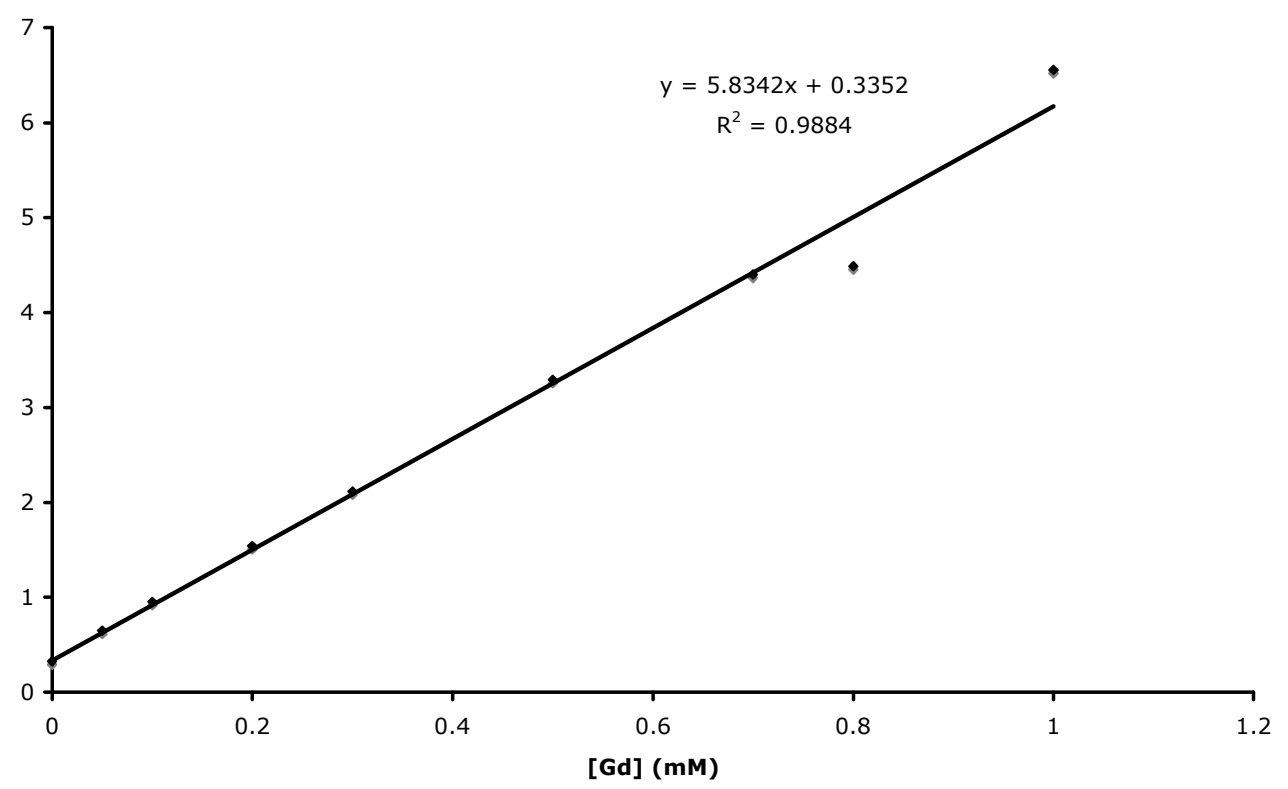


Relaxivity of Gd.3 in PBS (phosphate buffered saline) (500 $\mathrm{MH}_{\mathrm{z}}$ )

MW: $591.7 \mathrm{~g} / \mathrm{mol}$

\begin{tabular}{|l|l|l|}
\hline Concentration $(\mathrm{mM})$ & $\mathrm{T}_{1}(\mathrm{~s})$ & $1 / \mathrm{T}_{1}\left(\mathrm{~s}^{-1}\right)$ \\
\hline 0 & 2.9090 & 0.3438 \\
\hline 0.05 & 1.8010 & 0.5552 \\
\hline 0.1 & 1.2090 & 0.8271 \\
\hline 0.2 & 0.8274 & 1.2086 \\
\hline 0.3 & 0.6049 & 1.6531 \\
\hline 0.5 & 0.4055 & 2.4661 \\
\hline 0.7 & 0.2891 & 3.4590 \\
\hline 0.8 & 0.2473 & 4.0437 \\
\hline 1.0 & 0.2114 & 4.7304 \\
\hline
\end{tabular}

The relaxivity of Gd.3Re(bpy)OTf in $\mathrm{H}_{2} \mathrm{O}(500 \mathrm{MHz})$

MW: $1167.2 \mathrm{~g} / \mathrm{mol}$

\begin{tabular}{|l|l|l|}
\hline Concentration $(\mathrm{mM})$ & $\mathrm{T}_{1}(\mathrm{~s})$ & $1 / \mathrm{T}_{1}\left(\mathrm{~s}^{-1}\right)$ \\
\hline 0 & 2.5710 & 0.3810 \\
\hline 0.05 & 1.3870 & 0.7210 \\
\hline 0.1 & 0.9249 & 1.0812 \\
\hline 0.2 & 0.6050 & 1.6529 \\
\hline 0.3 & 0.3388 & 2.9516 \\
\hline 0.4 & 0.2610 & 3.8314 \\
\hline 0.5 & 0.2243 & 4.4583 \\
\hline 0.6 & 0.1810 & 5.5249 \\
\hline 0.7 & 0.1670 & 5.9880 \\
\hline 0.8 & 0.1400 & 7.1429 \\
\hline 0.9 & 0.1250 & 8.0000 \\
\hline 1.0 & 0.1110 & 9.0090 \\
\hline
\end{tabular}


The relaxivity of Gd.3Re(bpy)OTf in PBS (phosphate buffered saline) (500 MHz) MW: $1167.2 \mathrm{~g} / \mathrm{mol}$

\begin{tabular}{|l|l|l|}
\hline Concentration $(\mathrm{mM})$ & $\mathrm{T}_{1}(\mathrm{~s})$ & $1 / \mathrm{T}_{1}\left(\mathrm{~s}^{-1}\right)$ \\
\hline 0 & 2.5430 & 0.3932 \\
\hline 0.05 & 1.3880 & 0.7210 \\
\hline 0.1 & 1.058 & 0.9452 \\
\hline 0.2 & 0.6280 & 1.5923 \\
\hline 0.3 & 0.5002 & 1.9992 \\
\hline 0.4 & 0.4620 & 2.1645 \\
\hline 0.5 & 0.3535 & 2.8289 \\
\hline 0.6 & 0.3450 & 2.8986 \\
\hline 0.8 & 0.2740 & 3.6496 \\
\hline 0.9 & 0.2430 & 4.1152 \\
\hline
\end{tabular}

The relaxivity of GdRe(bpy) in PBS (phosphate buffered saline) (400 MHz) MW: $1017 \mathrm{~g} / \mathrm{mol}$

\begin{tabular}{|l|l|l|}
\hline Concentration $(\mathrm{mM})$ & $\mathrm{T}_{1}(\mathrm{~s})$ & $1 / \mathrm{T}_{1}\left(\mathrm{~s}^{-1}\right)$ \\
\hline 0 & 2.9326 & 0.3410 \\
\hline 0.1 & 2.1942 & 0.4558 \\
\hline 0.2 & 1.4092 & 0.7096 \\
\hline 0.5 & 0.8029 & 1.2455 \\
\hline 1.0 & 0.4462 & 2.2412 \\
\hline
\end{tabular}


1. Sheldrick, G. M.; SHELXTL 5.04, An integrated system for solving, refining and displaying crystal structures from diffraction data, Siemens Analytical X-ray Instruments Inc., Madison, WI, 1995.

PLATON, Speck, A. L. Acta Crystallogr. Sect. A 1990, 46, C34.

2. Dadabhoy, A.; Faulkner, S.; Sammes, P. G. J. Chem. Soc., Perkin Trans. 2 2002, 348-357.

3. Wrighton, M.; Morse, D. L. J. Am. Chem. Soc., 1974, 96, 998-357.

4. Lin, R.; Fu, Y.; Brock, C. P.; Guarr, T. F. Inorg. Chem., 1992, 31, 4346-4353. 